Andreas Čap

\title{
Infinitesimal automorphisms and deformations of parabolic geometries
}

Received November 21, 2005 and in revised form December 10, 2006

\begin{abstract}
We show that infinitesimal automorphisms and infinitesimal deformations of parabolic geometries can be nicely described in terms of the twisted de Rham sequence associated to a certain linear connection on the adjoint tractor bundle. For regular normal geometries, this description can be related to the underlying geometric structure using the machinery of BGG sequences. In the locally flat case, this leads to a deformation complex, which generalizes the well known complex for locally conformally flat manifolds.

Recently, a theory of subcomplexes in BGG sequences has been developed. This applies to certain types of torsion free parabolic geometries including quaternionic structures, quaternionic contact structures and CR structures. We show that for these structures one of the subcomplexes in the adjoint BGG sequence leads (even in the curved case) to a complex governing deformations in the subcategory of torsion free geometries. For quaternionic structures, this deformation complex is elliptic.
\end{abstract}

Keywords. Parabolic geometry, BGG sequence, quaternionic structure, quaternionic contact structure, CR structure, infinitesimal automorphism, infinitesimal deformation, deformation complex

\section{Introduction}

Given a smooth manifold $M$ and a type of geometric structure, it is a natural idea to consider the moduli space, i.e. the space of isomorphism classes of structures of the given type on $M$. This moduli space can be viewed as the quotient of the space of all structures of the given type by the action of the diffeomorphism group of $M$, which acts by pulling back structures. In general, the moduli space is a highly complicated object. Trying to understand the moduli space locally, one is led to the study of deformations of geometric structures. Here deformations coming from the action of one-parameter groups of diffeomorphisms have to be considered as trivial. Reducing further to the formal infinitesimal level, one arrives at infinitesimal deformations. These describe the possible directions in which a given structure can be deformed. As before, there is the notion of a trivial infinitesimal deformation, and the quotient of the space of all infinitesimal deformations by

A. Čap: Institut für Mathematik, Universität Wien, Nordbergstr. 15, A-1090 Wien, Austria, and International Erwin Schrödinger Institute for Mathematical Physics, Boltzmanngasse 9, A-1090 Wien, Austria; e-mail: Andreas.Cap@esi.ac.at

Mathematics Subject Classification (2000): 32V05, 53A40, 53B15, 53C15, 53D10, 58H15, 58J10 
the trivial ones is usually referred to as the formal tangent space of the moduli space at the given structure.

In this paper, we study infinitesimal deformations and the closely related infinitesimal automorphisms for parabolic geometries. These form a large class of geometric structures containing examples like conformal, quaternionic, hypersurface type CR, and certain higher codimension CR structures. For some of these structures, deformation theory has been developed quite far. Infinitesimal deformations are usually defined in an ad hoc manner as smooth sections of some bundle. Trivial infinitesimal deformations are those which lie in the image of some linear differential operator, whose kernel is the space of infinitesimal automorphisms. In particular, the formal tangent space is usually infinitedimensional.

It is a highly interesting problem to restrict the class of allowed deformations in such a way that one obtains a finite-dimensional moduli space. This can be done by imposing integrability conditions on the geometric structure and looking only at deformations in the subclass of geometries satisfying these conditions. For parabolic geometries, the simplest possible condition is local flatness. In some cases, much more subtle integrability conditions can be used, for example anti-self-duality for conformal structures in dimension four.

The unifying feature of parabolic geometries is that they can be viewed as Cartan geometries with homogeneous model a generalized flag manifold. Regular normal geometries of this type are then equivalent to underlying geometric structures including the examples listed above. For Cartan geometries, there are evident notions of infinitesimal deformations and infinitesimal automorphisms. These can be nicely formulated in terms of a certain linear connection (which surprisingly is different from the canonical normal tractor connection) on the adjoint tractor bundle (see Proposition 3.2). In particular, the relevant operators are part of the twisted de Rham sequence associated to this linear connection.

The machinery of Bernstein-Gelfand-Gelfand sequences (or BGG sequences), which was introduced in [13] and improved in [5], can be applied to this twisted de Rham sequence. One obtains a sequence of higher order operators acting on sections of bundles that can be easily interpreted in terms of the underlying structure. For regular normal geometries, the first operator in this sequence has the space of infinitesimal automorphisms as its kernel and the formal tangent space to the moduli space of normal geometries as its cokernel (see 3.4 and 3.6.

For locally flat parabolic geometries (which are automatically regular and normal, and locally isomorphic to the homogeneous model), the twisted de Rham sequence is a complex. Thus also the corresponding BGG sequence is a complex which can be naturally interpreted as a deformation complex in the category of locally flat structures.

Finally, we move to more subtle integrability conditions. The recent joint work [14] with V. Souček contains a theory of subcomplexes in BGG sequences. In that paper, we study several examples, in which there is an interesting notion of semiflatness which includes (and in most cases is equivalent to) torsion freeness. In particular, these include quaternionic structures and CR structures, but also quaternionic contact structures (torsion free ones in dimension 7) as introduced in [3, 4]. In Section 4, we show that for all these 
geometries a certain subcomplex of the adjoint BGG sequence can be naturally interpreted as a deformation complex in the subcategory of semiflat geometries. For quaternionic structures, this deformation complex is elliptic.

\section{Some background}

We very briefly review some background. Some more details can be found in [14] and much more information is available in [13, 11, 7, 12].

\subsection{Parabolic geometries}

The basic data needed to define a parabolic geometry is a semisimple Lie algebra $\mathfrak{g}$ endowed with a $|k|$-grading $\mathfrak{g}=\mathfrak{g}_{-k} \oplus \cdots \oplus \mathfrak{g}_{k}$ and a group $G$ with Lie algebra $\mathfrak{g}$. The subgroup $P \subset G$ consisting of all elements $g \in G$ such that $\operatorname{Ad}(g)\left(\mathfrak{g}^{i}\right) \subset \mathfrak{g}^{i}$ for all $i$, where $\mathfrak{g}^{i}:=\mathfrak{g}_{i} \oplus \cdots \oplus \mathfrak{g}_{k}$, is a parabolic subgroup. We will also need the subgroup $G_{0} \subset P$ of all elements whose adjoint action preserves the grading of $\mathfrak{g}$.

Parabolic geometries of type $(G, P)$ are then defined as Cartan geometries of that type. Such a geometry on a smooth manifold $M$ consists of a principal $P$-bundle $p$ : $\mathcal{G} \rightarrow M$ and a Cartan connection $\omega \in \Omega^{1}(\mathcal{G}, \mathfrak{g})$. The homogeneous model of parabolic geometries of type $(G, P)$ is given by the canonical principal bundle $G \rightarrow G / P$ with the left Maurer-Cartan form as a Cartan connection. A morphism of parabolic geometries is a homomorphism of principal bundles which is compatible with the Cartan connections. In particular, any morphism is a local diffeomorphism.

The curvature of a Cartan connection $\omega$ can be viewed as the two-form $K \in \Omega^{2}(\mathcal{G}, \mathfrak{g})$ defined by the structure equation

$$
K(\xi, \eta)=d \omega(\xi, \eta)+[\omega(\xi), \omega(\eta)],
$$

where $\xi$ and $\eta$ are vector fields on $\mathcal{G}$ and the bracket is in $\mathfrak{g}$. Since $K$ is horizontal and equivariant, it can be interpreted as a two-form $\kappa$ on $M$ with values in the associated bundle $\mathcal{A} M:=\mathcal{G} \times_{P} \mathfrak{g}$ (see 3.1 for more details). The bundle $\mathcal{A} M$ is called the adjoint tractor bundle. The $P$-invariant filtration $\left\{\mathfrak{g}^{i}\right\}$ of $\mathfrak{g}$ gives rise to a filtration $\mathcal{A} M=\mathcal{A}^{-k} M \supset \cdots \supset \mathcal{A}^{k} M$ by smooth subbundles and the Lie bracket on $\mathfrak{g}$ gives rise to a tensorial bracket $\{$,$\} on \mathcal{A} M$, making it into a bundle of filtered Lie algebras modeled on $\mathfrak{g}$.

On the other hand, the Cartan connection $\omega$ induces an isomorphism between the tangent bundle $T M$ and the associated bundle $\mathcal{G} \times P(\mathfrak{g} / \mathfrak{p})$. Hence there is a natural projection $\Pi: \mathcal{A} M \rightarrow \mathcal{T} M$ which induces an isomorphism $\mathcal{A} M / \mathcal{A}^{0} M \cong T M$. Via this isomorphism, the filtration of $\mathcal{A} M$ descends to a filtration $T M=T^{-k} M \supset \cdots \supset T^{-1} M$ of the tangent bundle by smooth subbundles.

Applying the projection $\Pi$ to the values of $\kappa$, we obtain a $T M$-valued two-form $\kappa_{-}$, which is called the torsion of the Cartan connection $\omega$. The geometry is called torsion free if this torsion vanishes.

Via the filtrations of $T M$ and $\mathcal{A} M$, one has a natural notion of homogeneity for $\mathcal{A} M$ valued differential forms. In particular, we say that $\kappa$ is homogeneous of degree $\geq \ell$ if 
$\kappa\left(T^{i} M, T^{j} M\right) \subset \mathcal{A}^{i+j+\ell} M$ for all $i, j=-k, \ldots,-1$. A parabolic geometry is called regular if its curvature is homogeneous of degree $\geq 1$. Note that torsion free parabolic geometries are automatically regular.

For parabolic geometries, there is a uniform normalization condition. This comes from the Kostant codifferential, which is the differential $\partial^{*}: \Lambda^{k} \mathfrak{p}_{+} \otimes \mathfrak{g} \rightarrow \Lambda^{k-1} \mathfrak{p}_{+} \otimes \mathfrak{g}$ in the standard complex computing Lie algebra homology of $\mathfrak{p}_{+}:=\mathfrak{g}_{1} \oplus \cdots \oplus \mathfrak{g}_{k}$ with coefficients in the representation $\mathfrak{g}$. Now $\mathfrak{p}_{+}$is dual to $\mathfrak{g} / \mathfrak{p}$ as a $P$-module via the Killing form, so $\mathcal{G} \times{ }_{P}\left(\Lambda^{k} \mathfrak{p}_{+} \otimes \mathfrak{g}\right) \cong \Lambda^{k} T^{*} M \otimes \mathcal{A} M$. Since $\partial^{*}$ is $P$-equivariant it induces a bundle map $\Lambda^{k} T^{*} M \otimes \mathcal{A} M \rightarrow \Lambda^{k-1} T^{*} M \otimes \mathcal{A} M$ as well as a tensorial operator $\Omega^{k}(M, \mathcal{A} M) \rightarrow \Omega^{k-1}(M, \mathcal{A} M)$, all denoted by $\partial^{*}$. A parabolic geometry is called normal if $\partial^{*}(\kappa)=0$.

Several important geometric structures like conformal structures, almost quaternionic structures, non-degenerate CR structures of hypersurface type, and quaternionic contact structures admit an equivalent description as a regular normal Cartan geometry of type $(G, P)$ for an appropriate choice of $(G, P)$. A description of this equivalence can be found in [7]. Thus parabolic geometries offer a powerful general machinery to study a variety of geometric structures.

\subsection{Bernstein-Gelfand-Gelfand sequences}

These are generalizations of the BGG resolutions from representation theory to sequences of invariant differential operators on parabolic geometries. They were introduced in [13] and the construction was improved in [5]. We will briefly sketch this improved construction for regular geometries in the special case of the adjoint tractor bundle; more details can be found in [14,6].

The Cartan connection $\omega$ induces a natural linear connection $\nabla$, called the adjoint tractor connection, on the adjoint tractor bundle $\mathcal{A M}$. This in turn induces the covariant exterior derivative

$$
d^{\nabla}: \Omega^{k}(M, \mathcal{A} M) \rightarrow \Omega^{k+1}(M, \mathcal{A} M) .
$$

The BGG machinery relates $d^{\nabla}$ to higher order operators acting on sections of certain subquotient bundles. Let $\partial^{*}: \Lambda^{k} T^{*} M \otimes \mathcal{A} M \rightarrow \Lambda^{k-1} T^{*} M \otimes \mathcal{A} M$ denote the bundle maps induced by the Kostant codifferential. The kernels and images of these bundle maps are natural subbundles, so we can look at the quotient bundles $\operatorname{ker}\left(\partial^{*}\right) / \operatorname{im}\left(\partial^{*}\right)$. By construction, they are associated to the representations $H_{k}\left(\mathfrak{p}_{+}, \mathfrak{g}\right)$. It turns out that the latter representations are always completely reducible and they are algorithmically computable using Kostant's version of the Bott-Borel-Weil theorem. Since the associated bundles can be viewed as the fiberwise homology groups of the bundle $T^{*} M$ of Lie algebras with coefficients in the bundle $\mathcal{A} M$, we denote them by $H_{k}\left(T^{*} M, \mathcal{A M}\right)$. Note that by construction there is a natural bundle map $\pi_{H}: \operatorname{ker}\left(\partial^{*}\right) \rightarrow H_{k}\left(T^{*} M, \mathcal{A M}\right)$, and we will denote by the same symbol the induced tensorial operator on sections.

For a normal parabolic geometry, the Cartan curvature $\kappa$ by definition is a section of $\operatorname{ker}\left(\partial^{*}\right)$, so we obtain the section $\kappa_{H}=\pi_{H}(\kappa)$ of the bundle $H_{2}\left(T^{*} M, \mathcal{A} M\right)$, which is called the harmonic curvature. This is a much simpler object than $\kappa$, but still a complete 
obstruction to local flatness. The components of $\kappa_{H}$ (according to the decomposition of $H_{2}\left(\mathfrak{p}_{+}, \mathfrak{g}\right)$ into irreducibles) are the fundamental invariants of a regular normal parabolic geometry.

The main step towards BGG sequences is the construction of a natural differential operator $L: \Gamma\left(H_{k}\left(T^{*} M, \mathcal{A} M\right)\right) \rightarrow \Omega^{k}(M, \mathcal{A} M)$, which admits a simple characterization. For $\alpha \in \Gamma\left(H_{k}\left(T^{*} M, \mathcal{A} M\right)\right)$ one has $\partial^{*}(L(\alpha))=0, \pi_{H}(L(\alpha))=\alpha$, and $\partial^{*}\left(d^{\nabla} L(\alpha)\right)=0$, and this determines $L$. In particular, we obtain a differential splitting of the tensorial projection $\pi_{H}: \Gamma\left(\operatorname{ker}\left(\partial^{*}\right)\right) \rightarrow \Gamma\left(H_{k}\left(T^{*} M, \mathcal{A} M\right)\right)$. Therefore, the operators $L$ are referred to as the splitting operators. The last property implies that we can define invariant differential operators by

$$
D:=\pi_{H} \circ d^{\nabla} \circ L: \Gamma\left(H_{k}\left(T^{*} M, \mathcal{A} M\right)\right) \rightarrow \Gamma\left(H_{k+1}\left(T^{*} M, \mathcal{A} M\right)\right),
$$

and these operators form the adjoint BGG sequence. Each of the bundles $H_{k}\left(T^{*} M, \mathcal{A M}\right)$ splits into a direct sum of subbundles according to the splitting of the representation $H_{*}\left(\mathfrak{p}_{+}, \mathfrak{g}\right)$ into irreducible components. Doing this in all degrees, one obtains a pattern of operators acting between the various components.

It is crucial for this article that this construction does not only work for $d^{\nabla}$. One only needs that the operator $\partial^{*} d^{\nabla}$ acts invertibly on $\Gamma\left(\operatorname{im}\left(\partial^{*}\right)\right) \subset \Omega^{k}(M, \mathcal{A} M)$ and the inverse is a differential operator. This in turn only requires that $d^{\nabla}$ preserves homogeneity of differential forms and the form of its component of homogeneous degree zero.

\subsection{Infinitesimal deformations of conformal structures}

For the convenience of the reader, we briefly review some basic results on infinitesimal deformations of conformal structures. Let $M$ be a smooth manifold of dimension $n \geq 3$ and let $[g]$ be a conformal class of pseudo-Riemannian metrics on $M$. An infinitesimal deformation of a pseudo-Riemannian metric is simply a smooth section $h$ of the bundle $S^{2} T^{*} M$. To obtain a deformation of the conformal class $[g]$ one first requires $h$ to be trace free. Second, one requires that if we rescale the metric $g$ in the conformal class, $h$ has to rescale in the same way. This means that $h$ has to be a section of the tensor product of $S_{0}^{2} T^{*} M$ with a certain density bundle. Using the notation and conventions of [9], the right bundle is $F_{1}:=S_{0}^{2} T^{*} M[2]=S_{0}^{2} T^{*} M \otimes \mathcal{E}[2]$.

Trivial deformations are those coming from pulling back the given structure along diffeomorphisms. The conformal class [ $g$ ] can be viewed as a section $\mathbf{g}$ of $S^{2} T^{*} M$ [2]. It turns out that for any vector field $\xi$, the Lie derivative $\mathcal{L}_{\xi} \mathbf{g}$ is a section of $F_{1}$, and these are the trivial infinitesimal deformations. Hence the quotient of all infinitesimal deformations by the trivial ones can be interpreted as the cokernel of the (by construction invariant) linear differential operator $D_{0}: \Gamma(T M) \rightarrow \Gamma\left(F_{1}\right)$ given by $D_{0}(\xi)=\mathcal{L}_{\xi} \mathbf{g}$. It is easy to verify that $D_{0}$ is the conformal Killing operator. In particular, its kernel is the space of conformal Killing fields, i.e. of infinitesimal conformal isometries of $(M,[g])$.

This is about how far one can get for general conformal structures. To proceed further one can impose some integrability condition on the conformal structure and look at deformations in the subclass of structures satisfying this condition. The simplest choice of such a condition is local conformal flatness. We will discuss this only for $n \geq 4$; the case $n=3$ 
is similar. For $n \geq 4$, local conformal flatness is equivalent to vanishing of the Weyl curvature. Hence it is natural to consider the bundle $F_{2}$, in which the Weyl curvature has its values, and the operator $D_{1}: \Gamma\left(F_{1}\right) \rightarrow \Gamma\left(F_{2}\right)$, which computes the infinitesimal change of the Weyl curvature caused by an infinitesimal deformation. If $(M,[g])$ is locally conformally flat, then sections in the kernel of $D_{1}$ correspond to infinitesimal deformations in the subcategory of locally conformally flat structures. Moreover, $D_{1} \circ D_{0}=0$ in that case, so the quotient $\operatorname{ker}\left(D_{1}\right) / \operatorname{im}\left(D_{0}\right)$ is exactly the formal tangent space to the moduli space of locally conformally flat structures on $M$.

It turns out that, still in the locally conformally flat case, this extends to a fine resolution

$$
0 \rightarrow \mathcal{K} \rightarrow \Gamma(T M) \stackrel{D_{0}}{\longrightarrow} \Gamma\left(F_{1}\right) \stackrel{D_{1}}{\longrightarrow} \Gamma\left(F_{2}\right) \stackrel{D_{2}}{\longrightarrow} \cdots \stackrel{D_{n-1}}{\longrightarrow} \Gamma\left(F_{n}\right) \rightarrow 0
$$

of the sheaf $\mathcal{K}$ of conformal Killing fields on $M$. Constructing this resolution by hand is fairly involved (see the book [15]).

In the case of four-dimensional conformal structures, a weaker integrability condition is available. In this case, the bundle $F_{2}$ splits into the direct sum $F_{2}^{+} \oplus F_{2}^{-}$of self-dual and anti-self-dual parts. Accordingly, the Weyl curvature splits as $W=W^{+}+W^{-}$, so $D_{1}=D_{1}^{+}+D_{1}^{-}$. Conformal structures with $W^{+}=0$ are called anti-self-dual. On such a structure $\operatorname{ker}\left(D_{1}^{+}\right)$exactly consists of infinitesimal deformations in the subcategory of anti-self-dual structures. It turns out that in this case

$$
0 \rightarrow \Gamma(T M) \stackrel{D_{0}}{\longrightarrow} \Gamma\left(S_{0}^{2} T^{*} M[2]\right) \stackrel{D_{1}^{+}}{\longrightarrow} \Gamma\left(F_{2}^{+}\right) \rightarrow 0
$$

is a complex, which is elliptic for Riemannian signature. This is the basis of the deformation theory for anti-self-dual conformal Riemannian four manifolds (see [17] and [16]).

\section{Infinitesimal automorphisms and deformations}

\subsection{The basic setup}

Fix a parabolic geometry ( $p: \mathcal{G} \rightarrow M, \omega)$ of some type $(G, P)$. By definition, the adjoint tractor bundle $\mathcal{A} M$ is the associated bundle $\mathcal{G} \times{ }_{P} \mathfrak{g}$ corresponding to the restriction of the adjoint representation of $G$ to $P$. Smooth sections of this bundle are in bijective correspondence with smooth functions $f: \mathcal{G} \rightarrow \mathfrak{g}$ such that $f(u \cdot g)=\operatorname{Ad}\left(g^{-1}\right)(f(u))$ for all $u \in \mathcal{G}$ and $g \in P$. More generally, for $k=1, \ldots, \operatorname{dim}(M)$ the space $\Omega^{k}(M, \mathcal{A} M)$ can be identified with the space $\Omega_{\text {hor }}^{k}(\mathcal{G}, \mathfrak{g})^{P}$ of $P$-equivariant, horizontal $\mathfrak{g}$-valued $k$-forms on $\mathcal{G}$. Here $\Phi \in \Omega^{k}(\mathcal{G}, \mathfrak{g})$ is horizontal if it vanishes upon insertion of one fundamental vector field, and $P$-equivariant if $\left(r^{g}\right)^{*} \Phi=\operatorname{Ad}\left(g^{-1}\right) \circ \Phi$ for all $g \in P$. Note that this identification is independent of the Cartan connection $\omega$.

This correspondence immediately leads to a geometric interpretation of $\Omega^{1}(M, \mathcal{A M})$ : Suppose that $\tilde{\omega} \in \Omega^{1}(\mathcal{G}, \mathfrak{g})$ is a second Cartan connection on $\mathcal{G}$. Then the difference $\tilde{\omega}-\omega \in \Omega^{1}(\mathcal{G}, \mathfrak{g})$ is by definition horizontal and $P$-equivariant, and thus corresponds to an element of $\Omega^{1}(M, \mathcal{A} M)$. There is an obvious notion of a deformation of the Cartan geometry $(\mathcal{G} \rightarrow M, \omega)$ as a smooth family $\omega_{\tau}$ of Cartan connections on $\mathcal{G}$ parametrized by $\tau \in(-\epsilon, \epsilon) \subset \mathbb{R}$ such that $\omega_{0}=\omega$. The initial direction of this deformation is 
the derivative $\left.\frac{d}{d \tau}\right|_{\tau=0} \omega_{\tau}$ of this family at $\tau=0$. By the above, this derivative can be interpreted as $\varphi \in \Omega^{1}(M, \mathcal{A} M)$.

Conversely, if $\Phi \in \Omega^{1}(\mathcal{G}, \mathfrak{g})$ is horizontal and $P$-equivariant, then $\omega+\Phi$ is a Cartan connection provided that it restricts to a linear isomorphism on each tangent space. Since this is an open condition, we can view $\Omega^{1}(M, \mathcal{A M})$ as the space of all directions of deformations of the Cartan connection $\omega$, i.e. as the space of all infinitesimal deformations of $\omega$.

From 2.1 we know that the curvature of any Cartan connection on $\mathcal{G}$ is naturally interpreted as an element of $\Omega^{2}(M, \mathcal{A} M)$. In particular, for a deformation $\omega_{\tau}$ of $\omega$, the resulting infinitesimal change of the curvature can be viewed as an element of $\Omega^{2}(M, \mathcal{A} M)$.

To discuss $\Omega^{0}(M, \mathcal{A} M)=\Gamma(\mathcal{A} M)$ we need a second interpretation of $C^{\infty}(\mathcal{G}, \mathfrak{g})^{P}$. Since $\omega$ trivializes $T \mathcal{G}$, associating to a vector field $\xi$ on $\mathcal{G}$ the function $\omega \circ \xi$ defines a bijection $\mathfrak{X}(\mathcal{G}) \rightarrow C^{\infty}(\mathcal{G}, \mathfrak{g})$. Equivariance of $\omega$ immediately implies that $(\omega \circ \xi) \circ r^{g}=$ $\operatorname{Ad}\left(g^{-1}\right) \circ(\omega \circ \xi)$ if and only if $\left(r^{g}\right)^{*} \xi=\xi$, so we obtain a bijection between $\Gamma(\mathcal{A} M)$ and the space $\mathfrak{X}(\mathcal{G})^{P}$ of $P$-invariant vector fields on $\mathcal{G}$. Notice that $P$-invariant vector fields are automatically projectable to vector fields on $M$, and this corresponds to the projection $\Pi: \mathcal{A} M \rightarrow T M$ from 2.1

A vector field $\xi \in \mathfrak{X}(\mathcal{G})$ satisfies $\left(r^{g}\right)^{*} \xi=\xi$ if and only if its flow commutes with $r^{g}$, whenever the flow is defined. This is true for all $g \in P$ if and only if the local flows are principal bundle automorphisms. Thus we can view the space $\Gamma(\mathcal{A} M)$ as the space of infinitesimal principal bundle automorphisms of the Cartan bundle $\mathcal{G}$.

\subsection{Formulae in the Cartan picture}

Given a section of $\mathcal{A M}$, we can look at the corresponding vector field on $\mathcal{G}$. The local flows of this vector field are principal bundle automorphisms, so we can use them to pull back the Cartan connection $\omega$, which locally defines a deformation of $\omega$. Deformations obtained in this way and also the corresponding infinitesimal deformations are called trivial. Note that while flows may be only locally defined, the corresponding infinitesimal deformation is always defined globally.

An automorphism of the parabolic geometry $(\mathcal{G}, \omega)$ by definition is a principal bundle automorphism $\Phi$ of $\mathcal{G}$ such that $\Phi^{*} \omega=\omega$. Correspondingly, an infinitesimal automorphism is a $P$-invariant vector field $\xi$ on $\mathcal{G}$ such that the induced infinitesimal deformation of the Cartan connection vanishes identically.

In studying the infinitesimal change of curvature caused by an infinitesimal deformation of the Cartan connection, there is an additional subtlety. For a deformation $\omega_{\tau}$ of $\omega=\omega_{0}$, we may view the curvature $\kappa_{\tau}$ of $\omega_{\tau}$ as an element of $\Omega^{2}(M, \mathcal{A M})$, and we could simply differentiate this family of sections. However, the identification of $\Lambda^{2} T^{*} M \otimes \mathcal{A} M$ with the associated bundle $\mathcal{G} \times_{P} \Lambda^{2} \mathfrak{p}_{+} \otimes \mathfrak{g}$, which is used to construct operators acting on the curvature, depends on the Cartan connection. The easiest way to take this into account is to first convert $\kappa_{\tau}$ into an equivariant function $\mathcal{G} \rightarrow \Lambda^{2} \mathfrak{p}_{+} \otimes \mathfrak{g}$ using $\omega_{\tau}$. Then one takes the derivative of this family of functions at $\tau=0$ and converts it back to an element of $\Omega^{2}(M, \mathcal{A} M)$ using $\omega=\omega_{0}$. 
Finally, observe that by means of the projection $\Pi: \mathcal{A M} \rightarrow T M$, any section of $\mathcal{A} M$ has an underlying vector field on $M$. In particular, for $s \in \Gamma(\mathcal{A} M)$ we can insert $\Pi(s)$ into a (bundle-valued) differential form on $M$, and we write $i_{s}$ for the corresponding insertion operator. More generally, for $\varphi \in \Omega^{\ell}(M, \mathcal{A} M)$ and a vector bundle $V \rightarrow M$, we obtain an insertion operator $i_{\varphi}: \Omega^{k}(M, V) \rightarrow \Omega^{k+\ell-1}(M, V)$.

Proposition. Let $(\mathcal{G} \rightarrow M, \omega)$ be a parabolic geometry with curvature $\kappa \in \Omega^{2}(M, \mathcal{A} M)$. Let $\nabla$ be the adjoint tractor connection, and let $d^{\nabla}: \Omega^{k}(M, \mathcal{A} M) \rightarrow \Omega^{k+1}(M, \mathcal{A} M)$ be the corresponding covariant exterior derivative. Then we have:

(1) For $s \in \Gamma(\mathcal{A} M)$, the infinitesimal deformation of $\omega$ induced by the corresponding invariant vector field is given by $\nabla s+i_{s} \kappa$. In particular, $s$ is an infinitesimal automorphism if and only if $\nabla s=-i_{s} \kappa$.

(2) For an infinitesimal deformation $\varphi \in \Omega^{1}(M, \mathcal{A M})$ of the Cartan connection $\omega$, the induced infinitesimal change of the curvature is given by $d^{\nabla} \varphi-i_{\varphi} \kappa \in \Omega^{2}(M, \mathcal{A} M)$.

Proof. (1) Let $\xi \in \mathfrak{X}(\mathcal{G})^{P}$ be the vector field corresponding to $s$. Pulling back $\omega$ by its flow and taking the derivative, we get the Lie derivative $\mathcal{L}_{\xi} \omega \in \Omega^{1}(\mathcal{G}, \mathfrak{g})$. Using Cartan's formula and the definition of curvature we obtain

$$
\left(\mathcal{L}_{\xi} \omega\right)(\eta)=d\left(i_{\xi} \omega\right)(\eta)+d \omega(\xi, \eta)=\eta \cdot \omega(\xi)+\kappa(\xi, \eta)-[\omega(\xi), \omega(\eta)] .
$$

To ensure vanishing of $\mathcal{L}_{\xi} \omega$, it suffices to insert vector fields $\eta$ which are projectable to $M$. Let $\eta \in \mathfrak{X}(M)$ be the image of $\eta$. Then $\eta \cdot \omega(\xi)+[\omega(\eta), \omega(\xi)]$ represents the section $\nabla_{\underline{\eta}} \bar{s}$ (see [8, 2.7]), and the result follows.

(2) Let $\omega_{\tau}$ be a deformation of $\omega$, put $\Phi:=\left.\frac{d}{d \tau}\right|_{\tau=0} \omega_{\tau} \in \Omega_{\text {hor }}^{1}(\mathcal{G}, \mathfrak{g})^{P}$, and let $\varphi \in$ $\Omega^{1}(M, \mathcal{A} M)$ be the corresponding element. Viewed as $K_{\tau} \in \Omega^{2}(\mathcal{G}, \mathfrak{g})$, the curvature of $\omega_{\tau}$ is given by

$$
K_{\tau}(\xi, \eta)=d \omega_{\tau}(\xi, \eta)+\left[\omega_{\tau}(\xi), \omega_{\tau}(\eta)\right] .
$$

Differentiating with respect to $\tau$ at $\tau=0$ we obtain

$$
d \Phi(\xi, \eta)+[\Phi(\xi), \omega(\eta)]+[\omega(\xi), \Phi(\eta)] .
$$

Choose $\xi$ and $\eta$ to be $P$-invariant and denote by $s$ and $t$ the corresponding sections of $\mathcal{A} M$. Expanding the exterior derivative and using the fact that $\xi \cdot \Phi(\eta)+[\omega(\xi), \Phi(\eta)]$ represents $\nabla_{\Pi(s)}(\varphi(t))$, and likewise for the other term, we exactly obtain the formula for the covariant exterior derivative $d^{\nabla} \varphi(\Pi(s), \Pi(t))$.

As discussed above, we should however first convert $K_{\tau}$ into a function using $\omega_{\tau}$, which means looking at $K_{\tau}\left(\omega_{\tau}^{-1}(X), \omega_{\tau}^{-1}(Y)\right)$ for $X, Y \in \mathfrak{g}$, differentiate, and then convert the result back into a form using $\omega$. Differentiating the equation $X=\omega_{\tau}\left(\omega_{\tau}^{-1}(X)\right)$ we see that

$$
\left.\frac{d}{d \tau}\right|_{\tau=0} \omega_{\tau}^{-1}(X)=-\omega^{-1}\left(\Phi\left(\omega^{-1}(X)\right)\right) .
$$

To get the expression for the change of the curvature, we thus have to add to $d^{\nabla} \varphi$ the terms

$$
-K_{0}\left(\omega^{-1}\left(\Phi\left(\omega^{-1}(X)\right)\right), \omega^{-1}(Y)\right)-K_{0}\left(\omega^{-1}(X), \omega^{-1}\left(\Phi\left(\omega^{-1}(Y)\right)\right)\right),
$$

which exactly represent $-i_{\varphi} \kappa$. 
Remark. We consider infinitesimal automorphisms and deformations on the level of the total space of the Cartan bundle here. As discussed in 2.1, regular normal parabolic geometries are equivalent to underlying structures. For several of these structures, notions of infinitesimal automorphisms and deformations are available in the literature (see 2.3 for a sketch of the conformal case).

For infinitesimal automorphisms, it is easy to see that the two concepts are equivalent: The construction of the canonical normal Cartan connection induces an equivalence of categories between regular normal parabolic geometries and underlying structures. An automorphism of the underlying structure uniquely lifts to an automorphism of the parabolic geometry, and conversely any automorphism of a parabolic geometry induces an automorphism of the underlying structure on the base. Applying this to local flows of vector fields, one immediately concludes that there is a bijective correspondence between infinitesimal automorphisms in the two senses. We shall see below that this correspondence is implemented by the machinery of BGG sequences.

In the case of infinitesimal deformations the question is a bit more subtle, but the concepts still coincide in all cases that I am aware of. The basic point here is the following: The underlying structures of parabolic geometries can all be encoded as infinitesimal flag structures (see [11]). These are principal $G_{0}$-bundles endowed with certain partially defined differential forms. A small deformation of the underlying structure cannot change the isomorphism type of the principal bundle, so it can be viewed as a deformation of the partially defined differential forms. Since the subgroup $P_{+} \subset P$ is always contractible, the total space of the Cartan bundle must be a trivial $P_{+}$-principal bundle over the underlying $G_{0}$-bundle. Making choices, one can extend the partially defined differential forms from above to a Cartan connection of the principal $P$-bundle, and this transforms smooth families to smooth families. The canonical Cartan connection can then be constructed by a normalization process which again maps smooth families to smooth families. This construction will be described in detail in [12]. In this way, any deformation of the underlying structure gives rise to a deformation of the parabolic geometry, and since the converse direction is obvious, this establishes the equivalence of the two notions. We shall see in the examples below that this correspondence is implemented by the BGG machinery.

\subsection{A variant of the adjoint $B G G$ sequence}

Proposition 3.2 suggests considering the linear connection $\tilde{\nabla}$ on the bundle $\mathcal{A} M$ which is defined by $\nabla s=\nabla s+i_{s} \kappa$. To describe it we need some natural operators on adjoint tractors. The algebraic bracket $\{$,$\} is induced by the bracket on \mathfrak{g}$, while the Lie bracket [, ] is induced from the Lie bracket on $\mathfrak{X}(\mathcal{G})$. Finally, the fundamental derivative was introduced under the name of "fundamental $D$-operator" in Section 3 of [8].

Lemma. (1) For $\varphi \in \Omega^{k}(M, \mathcal{A} M)$ we have $d^{\tilde{\nabla}} \varphi=d^{\nabla} \varphi+(-1)^{k} i_{\varphi} \kappa$.

(2) The curvature $\tilde{R}$ of $\tilde{\nabla}$ is given by $\tilde{R}(\xi, \eta)(s)=\left(D_{s} \kappa\right)(\xi, \eta)$, where $D_{s}$ denotes the fundamental derivative. 
Proof. (1) is a straightforward computation using the standard formula

$$
\begin{aligned}
\left(d^{\tilde{\nabla}} \varphi\right)\left(\xi_{0}, \ldots, \xi_{k}\right)= & \sum_{i}(-1)^{i} \tilde{\nabla}_{\xi_{i}}\left(\varphi\left(\xi_{0}, \ldots, \hat{i}, \ldots, \xi_{k}\right)\right) \\
& +\sum_{i<j}(-1)^{i+j} \varphi\left(\left[\xi_{i}, \xi_{j}\right], \xi_{0}, \ldots, \hat{i}, \ldots, \hat{j}, \ldots, \xi_{k}\right)
\end{aligned}
$$

for the covariant exterior derivative.

(2) The action of $\tilde{R}$ on $s \in \Gamma(\mathcal{A} M)$ can be computed as $d^{\tilde{\nabla}} \tilde{\nabla} s$. Inserting the definition of $\tilde{\nabla}$ and using (1) shows that this equals $d^{\nabla} \nabla s+d^{\nabla}\left(i_{s} \kappa\right)-i_{\tilde{\nabla} s} \kappa$. The first term gives the action $\kappa \bullet s$ of the curvature of $\nabla$, i.e. $(\kappa \bullet s)(\xi, \eta)=\{\kappa(\xi, \eta), s\}$. Since $\kappa$ is the curvature of $\nabla$, the Bianchi identity for linear connections implies that $0=d^{\nabla} \kappa$. Taking $t_{1}, t_{2} \in \Gamma(\mathcal{A} M)$ and expanding $0=d^{\nabla} \kappa\left(t_{1}, s, t_{2}\right)$ we obtain the formula

$$
d^{\nabla}\left(i_{s} \kappa\right)\left(t_{1}, t_{2}\right)=\nabla_{s}\left(\kappa\left(t_{1}, t_{2}\right)\right)-\kappa\left(\left[s, t_{1}\right], t_{2}\right)-\kappa\left(t_{1},\left[s, t_{2}\right]\right) .
$$

Now $\nabla_{s}\left(\kappa\left(t_{1}, t_{2}\right)\right)=D_{s}\left(\kappa\left(t_{1}, t_{2}\right)\right)+\left\{s, \kappa\left(t_{1}, t_{2}\right)\right\}$ by [8, Proposition 3.2], while [8, Proposition 3.6] reads as $\left[s, t_{1}\right]=D_{s} t_{1}-\tilde{\nabla}_{t_{1}} s$. Inserting all these into the above formula for $d^{\tilde{\nabla}} \tilde{\nabla} s$ yields the claim.

Using part (1), we conclude from Proposition 3.2 that the infinitesimal change of curvature caused by an infinitesimal deformation of a Cartan connection is computed by $d^{\tilde{\nabla}}$.

Now suppose that we are dealing with a regular parabolic geometry $(p: \mathcal{G} \rightarrow M, \omega)$. By definition, this means that $\kappa$ is homogeneous of degree $\geq 1$, i.e. for $\xi \in \Gamma\left(T^{i} M\right)$ and $\eta \in \Gamma\left(T^{j} M\right)$, we have $\kappa(\xi, \eta) \in \Gamma\left(\mathcal{A}^{i+j+1} M\right)$. If $\varphi \in \bar{\Omega}^{k}(M, \mathcal{A} M)$ is homogeneous of degree $\geq \ell$, this immediately implies that $i_{\varphi} \kappa$ is homogeneous of degree $\geq \ell+1$. Therefore $d^{\tilde{\nabla}} \varphi$ is congruent to $d^{\nabla} \varphi$ modulo elements which are homogeneous of degree $\geq \ell+1$. Hence the lowest possibly nonzero homogeneous components of $d^{\nabla} \varphi$ and of $d^{\tilde{\nabla}} \varphi$ coincide. As pointed out in 2.2, this is all we need to apply the BGG machinery to the twisted de Rham sequence induced by $\tilde{\nabla}$.

We write $\tilde{L}: \Gamma\left(H_{k}\left(T^{*} M, \mathcal{A} M\right)\right) \rightarrow \Omega^{k}(M, \mathcal{A} M)$ for the splitting operators obtained by this construction. They are characterized by $\partial^{*}(\tilde{L}(\alpha))=0, \pi_{H}(\tilde{L}(\alpha))=\alpha$, and $\partial^{*}\left(d^{\tilde{\nabla}} \tilde{L}(\alpha)\right)=0$. The induced BGG operators $\tilde{D}_{k}: \Gamma\left(H_{k}\left(T^{*} M, \mathcal{A} M\right)\right) \rightarrow$ $\left.\Gamma\left(H_{k+1}\left(T^{*} M, \mathcal{A} M\right)\right)\right)$ are given by $\tilde{D}_{k}=\pi_{H} \circ d^{\tilde{\nabla}} \circ \tilde{L}$.

\subsection{Infinitesimal automorphisms}

It is easy to relate the BGG sequence obtained from $d^{\tilde{\nabla}}$ to infinitesimal automorphisms:

Theorem. Let $(p: \mathcal{G} \rightarrow M, \omega)$ be a regular normal parabolic geometry of type $(G, P)$ corresponding to a $|k|$-grading of $\mathfrak{g}$. Then the bundle $H_{0}\left(T^{*} M, \mathcal{A} M\right)$ equals $\mathcal{A} M / \mathcal{A}^{-k+1} M \cong T M / T^{-k+1} M$. The algebraic projection $\pi_{H}$ and the differential operator $\tilde{L}$ restrict to mutually inverse bijections between infinitesimal automorphisms of $(p: \mathcal{G} \rightarrow M, \omega)$ and smooth sections $\sigma \in \Gamma\left(T M / T^{-k+1} M\right)$ such that $\tilde{D}_{0}(\sigma)=0$. 
Proof. The bundle $H_{0}\left(T^{*} M, \mathcal{A M}\right)$ corresponds to the representation $H_{0}\left(\mathfrak{p}_{+}, \mathfrak{g}\right)$. By definition, this homology group is $\mathfrak{g} /\left[\mathfrak{p}_{+}, \mathfrak{g}\right]$, and it is well known that $\left[\mathfrak{p}_{+}, \mathfrak{g}\right]=\mathfrak{g}^{-k+1}$, so the statement about $H_{0}\left(T^{*} M, \mathcal{A} M\right)$ follows.

By Proposition 3.2 (1), a smooth section $s \in \Gamma(\mathcal{A} M)$ defines an infinitesimal automorphism if and only if $\tilde{\nabla} s=0$. If this is the case, then $\partial^{*}(\tilde{\nabla} s)=0$, and since $\partial^{*}(s)=0$ is automatically satisfied, this implies $s=\tilde{L}\left(\pi_{H}(s)\right)$ and $\tilde{D}_{0}\left(\pi_{H}(s)\right)=0$. Hence $\pi_{H}$ restricts to an injection from infinitesimal automorphisms to $\operatorname{ker}\left(\tilde{D}_{0}\right)$.

Conversely, if $\sigma \in \Gamma\left(T M / T^{-k+1} M\right)$ satisfies $\tilde{D}_{0}(\sigma)=0$, then put $s:=\tilde{L}(\sigma)$. Then $\partial^{*}(\tilde{\nabla} s)=0$ and $\tilde{D}_{0}(\sigma)=0$ implies that $\pi_{H}(\tilde{\nabla} s)=0$, so $\tilde{\nabla} s$ is a section of the subbundle $\operatorname{im}\left(\partial^{*}\right) \subset T^{*} M \otimes \mathcal{A} M$. By Proposition 3.2(2), we get $d^{\tilde{\nabla}} \tilde{\nabla} s=D_{s} \kappa$, and by naturality of the fundamental derivative and normality we get $\partial^{*}\left(D_{s} \kappa\right)=D_{s} \partial^{*}(\kappa)=0$. But from 2.2 we know that $\partial^{*} \circ d^{\tilde{\nabla}}$ is injective on sections of $\operatorname{im}\left(\partial^{*}\right)$, so $\tilde{\nabla} s=0$ and $s$ is an infinitesimal automorphism.

\subsection{Comparing the BGG operators}

To complete the discussion of infinitesimal automorphisms, it remains to compare the first operator $\tilde{D}_{0}$ in the BGG sequence associated to $\tilde{\nabla}$ with the first operator $D_{0}$ in the BGG sequence associated to $\nabla$.

Theorem. Let $(p: \mathcal{G} \rightarrow M, \omega)$ be a regular normal parabolic geometry of type $(G, P)$, and let $\mathfrak{g}$ be the Lie algebra of $G$. Let $L$ and $\tilde{L}$ be the splitting operators in degree zero and $D_{0}$ and $\tilde{D}_{0}$ the $B G G$ operators obtained from $\nabla$ and $\tilde{\nabla}$, respectively.

(1) If $\mathfrak{g}$ is $|1|$-graded or $(p: \mathcal{G} \rightarrow M, \omega)$ is torsion free, then

$$
L=\tilde{L}: \Gamma\left(T M / T^{-k+1} M\right) \rightarrow \Gamma(\mathcal{A} M) \quad \text { and } \quad \tilde{D}_{0}(\sigma)=D_{0}(\sigma)+\pi_{H}\left(i_{L(\sigma)} \kappa\right) .
$$

(2) If $(p: \mathcal{G} \rightarrow M, \omega)$ is torsion free and $H_{1}\left(\mathfrak{p}_{+}, \mathfrak{g}\right)$ is concentrated in non-positive homogeneity degrees then $\tilde{D}_{0}=D_{0}$.

Proof. (1) We start by computing $\partial^{*}\left(i_{\xi} \kappa\right)$ for an arbitrary vector field $\xi \in \mathfrak{X}(M)$. Locally, we can write $\kappa$ as a finite sum of terms of the form $\varphi \wedge \psi \otimes t$ for $\varphi, \psi \in \Omega^{1}(M)$ and $t \in \Gamma(\mathcal{A} M)$. By definition, $\partial^{*}(\kappa)$ is then the sum of the corresponding terms of the form

$$
-\psi \otimes\{\varphi, t\}+\varphi \otimes\{\psi, t\}-\{\varphi, \psi\} \otimes t .
$$

On the other hand, $i_{\xi} \kappa$ is the sum of the terms $\varphi(\xi) \psi \otimes t-\psi(\xi) \varphi \otimes t$. Thus $\partial^{*}\left(i_{\xi} \kappa\right)$ is the sum of the terms $\varphi(\xi)\{\psi, t\}-\psi(\xi)\{\varphi, t\}$, and we conclude that

$$
\partial^{*}\left(i_{\xi} \kappa\right)=-i_{\xi}\left(\partial^{*}(\kappa)-(\{,\} \otimes \mathrm{id})(\kappa)\right),
$$

where we use $\{,\} \otimes$ id $: \Lambda^{2} T^{*} M \otimes \mathcal{A} M \rightarrow T^{*} M \otimes \mathcal{A} M$. Since we are dealing with a normal parabolic geometry, we have $\partial^{*}(\kappa)=0$. In the case of a $|1|$-grading the map $\{\}:, \Lambda^{2} T^{*} M \rightarrow T^{*} M$ is identically zero, so we get $\partial^{*}\left(i_{\xi} \kappa\right)=0$ in this case.

In the torsion free case, we first observe that the kernel of $[,] \otimes$ id is a $P$-submodule in $\Lambda^{2} \mathfrak{p}_{+} \otimes \mathfrak{g}$. For any normal parabolic geometry, the harmonic curvature $\kappa_{H}=\pi_{H}(\kappa)$ has values in $H_{2}\left(T^{*} M, \mathcal{A M}\right)$. By Kostant's version of the Bott-Borel-Weyl theorem (see 
[18]) the corresponding subrepresentation has multiplicity one in $\Lambda^{*} \mathfrak{p}_{+} \otimes \mathfrak{g}$. In particular, it has to be contained in the kernel of $[,] \otimes$ id. By [6. Theorem 3.2(1)] the curvature of any torsion free parabolic geometry therefore has values in the kernel of $\{,\} \otimes$ id, so we again conclude that $\partial^{*}\left(i_{\xi} \kappa\right)=0$ for each $\xi$.

For a section $\sigma$ of $T M / T^{-k+1} M$, consider $L(\sigma)$. By construction, $\partial^{*}(L(\sigma))=0$, $\pi_{H}(L(\sigma))=\sigma$, and $\partial^{*}(\nabla L(\sigma))=0$. Since $\tilde{\nabla} L(\sigma)=\nabla L(\sigma)+i_{L(\sigma)} \kappa$, we also have $\partial^{*}(\tilde{\nabla} L(\sigma))=0$. Hence $L(\sigma)$ satisfies the three conditions which characterize $\tilde{L}(\sigma)$ and so $\tilde{L}=L$. Using this we obtain

$$
\tilde{D}_{0}(\sigma)=\pi_{H}(\tilde{\nabla} L(\sigma))=D_{0}(\sigma)+\pi_{H}\left(i_{L(\sigma)} \kappa\right) .
$$

(2) Since we are dealing with a torsion free geometry, we get $i_{s} \kappa \in \Omega^{1}\left(M, \mathcal{A}^{0} M\right) \subset$ $\Omega^{1}(M, \mathcal{A} M)$ for each $s \in \Gamma(\mathcal{A} M)$. In particular, $i_{s} \kappa$ is always homogeneous of degree $\geq 1$, so by the assumption on $H_{1}\left(\mathfrak{p}_{+}, \mathfrak{g}\right)$ we get $\pi_{H}\left(i_{L(\sigma)} \kappa\right)=0$ for any section $\sigma$ of $T M / T^{-k+1} M$.

Corollary. Suppose that $(p: \mathcal{G} \rightarrow M, \omega)$ is torsion free, $H_{1}\left(\mathfrak{p}_{+}, \mathfrak{g}\right)$ is concentrated in non-positive homogeneity degrees, and $s \in \Gamma(\mathcal{A} M)$ satisfies $\nabla s=0$. Then $i_{s} \kappa=0$ and in particular $s$ is an infinitesimal automorphism.

Proof. Since $\nabla s=0$ we get $s=L\left(\pi_{H}(s)\right)$ and $D_{0}\left(\pi_{H}(s)\right)=0$. By the theorem, we have $L=\tilde{L}$ and $D_{0}=\tilde{D}_{0}$, and in the proof of Theorem 3.4. we have seen that $\tilde{D}_{0}\left(\pi_{H}(s)\right)=0$ implies $\tilde{\nabla} s=0$.

Remark. (1) The condition that $H_{1}\left(\mathfrak{p}_{+}, \mathfrak{g}\right)$ is concentrated in non-positive homogeneity degrees is easy to verify (see [19] or [10, Proposition 2.7]): The semisimple $|k|$-graded Lie algebra $\mathfrak{g}$ decomposes as a direct sum of $\left|k_{i}\right|$-graded simple ideals with $k_{i} \leq k$ for each $i$. The condition is equivalent to the fact that none of these simple ideals is of type $A_{\ell}$ or $C_{\ell}$ with the grading corresponding to the first simple root. If $\mathfrak{g}$ itself is simple, then this exactly excludes classical projective structures and a contact analog of these. Note that in the latter two cases regular normal parabolic geometries are automatically torsion free, so part (1) of the theorem holds for all regular normal geometries of these types.

(2) The statement of the corollary is rather surprising even in special cases like conformal structures. The identities responsible for its validity are contained in the proof of Lemma 3.3. From this proof one easily deduces $d^{\nabla}\left(i_{s} \kappa\right)=D_{s} \kappa-\kappa \bullet s+i_{\tilde{\nabla} s} \kappa$ for any $s \in \Gamma(\mathcal{A} M)$. If $\nabla s=0$, then $0=d^{\nabla}(\nabla s)=\kappa \bullet s$ and if the geometry is torsion free then this also implies that $\tilde{\nabla} s$ has values in $\mathcal{A}^{0} M$ and hence $i_{\tilde{\nabla} s} \kappa=0$. Since $0=D_{s} \partial^{*}(\kappa)=\partial^{*}\left(D_{s} \kappa\right)$ we obtain $\partial^{*} d^{\nabla}\left(i_{s} \kappa\right)=0$, which under the assumptions of the corollary implies $i_{s} \kappa=0$.

\subsection{Infinitesimal deformations}

Consider an infinitesimal deformation $\varphi \in \Omega^{1}(M, \mathcal{A} M)$ of a regular normal parabolic geometry $(p: \mathcal{G} \rightarrow M, \omega)$. Then $\varphi$ is called normal if the deformed curvature (infinitesimally) remains normal. According to Propositions 3.2 and 3.3 , this is the case if and only if $\partial^{*}\left(d^{\tilde{\nabla}} \varphi\right)=0$. 
The BGG machinery now easily implies that the operator $\tilde{D}_{0}$ whose kernel is the space of infinitesimal automorphisms, also has the formal tangent space to the moduli space of normal geometries as its cokernel:

Theorem. Let $(p: \mathcal{G} \rightarrow M, \omega)$ be a regular normal parabolic geometry.

(1) Any trivial infinitesimal deformation of $\omega$ is normal.

(2) The splitting operator $\tilde{L}: \Gamma\left(H_{1}\left(T^{*} M, \mathcal{A} M\right)\right) \rightarrow \Omega^{1}(M, \mathcal{A} M)$ induces a bijection between $\Gamma\left(H_{1}\left(T^{*} M, \mathcal{A} M\right)\right) / \operatorname{im}\left(\tilde{D}_{0}\right)$ and the formal tangent space at the given structure to the moduli space of all normal parabolic geometries on $M$.

(3) The BGG operator $\tilde{D}_{1}$ computes the infinitesimal change of the harmonic curvature caused by the infinitesimal deformation $\tilde{L}(\alpha)$ associated to $\alpha \in \Gamma\left(H_{1}\left(T^{*} M, \mathcal{A M}\right)\right)$.

Proof. We have already observed in the proof of Theorem 3.4 that $d^{\tilde{\nabla}} \tilde{\nabla} s=D_{s} \kappa$ and that this has values in the kernel of $\partial^{*}$, so (1) follows.

For $\alpha \in \Gamma\left(H_{1}\left(T^{*} M, \mathcal{A} M\right)\right)$ we put $\varphi:=\tilde{L}(\alpha)$. Then by construction $\partial^{*}\left(d^{\tilde{\nabla}} \varphi\right)=0$, so $\varphi$ defines a normal infinitesimal deformation. By Proposition $3.2, d^{\tilde{\nabla}} \varphi$ is the infinitesimal change of curvature caused by $\varphi$, and by definition $\tilde{D}_{1}(\alpha)=\pi_{H}\left(d^{\tilde{\nabla}} \varphi\right)$, which implies (3).

If $\alpha=\tilde{D}_{0}(\sigma)$, then put $s=\tilde{L}(\sigma)$, so $\alpha=\pi_{H}(\tilde{\nabla} s)$. Since $\partial^{*}(\tilde{\nabla} s)=0$ and $\partial^{*}\left(d^{\tilde{\nabla}} \tilde{\nabla} s\right)=0$ we conclude that $\tilde{\nabla} s=\tilde{L}(\alpha)$, so the resulting deformation is trivial. Thus $\tilde{L}$ induces a map from the quotient $\Gamma\left(H_{1}\left(T^{*} M, \mathcal{A M}\right)\right) / \operatorname{im}\left(\tilde{D}_{0}\right)$ to normal infinitesimal deformations modulo trivial infinitesimal deformations.

Suppose that $\tilde{L}(\alpha)=\tilde{\nabla} s$. Then in particular $\partial^{*}(\tilde{\nabla} s)=0$, so $s=\tilde{L}\left(\pi_{H}(s)\right)$. Hence $\alpha=\tilde{D}_{0}\left(\pi_{H}(s)\right)$ and our map is injective. To prove surjectivity, suppose that $\varphi \in \Omega^{1}(M, \mathcal{A} M)$ is any normal infinitesimal deformation. Put $s=-\tilde{Q} \partial^{*}(\varphi)$, where $\tilde{Q}: \Gamma\left(\operatorname{im}\left(\partial^{*}\right)\right) \rightarrow \Gamma\left(\operatorname{im}\left(\partial^{*}\right)\right)$ is the inverse of $\partial^{*} \circ d^{\tilde{\nabla}}$ (cf. 2.2. . Replacing $\varphi$ by the equivalent infinitesimal deformation $\psi=\varphi+\tilde{\nabla} s$, we see that $\partial^{*}(\psi)=0$ and $\partial^{*}\left(d^{\tilde{\nabla}} \psi\right)=0$, so $\psi=\tilde{L}\left(\pi_{H}(\psi)\right)$ and surjectivity follows.

The relation between the splitting operators and the BGG operators obtained from $d^{\nabla}$ respectively $d^{\tilde{\nabla}}$ is much more complicated than for the first operator in the sequence. We just prove a simple general result here which is sufficient to deal with the cases discussed in this paper.

Lemma. Let $(p: \mathcal{G} \rightarrow M, \omega)$ be a torsion free normal parabolic geometry. Suppose that $V \subset H_{k}\left(\mathfrak{p}_{+}, \mathfrak{g}\right)$ and $W \subset H_{k+1}\left(\mathfrak{p}_{+}, \mathfrak{g}\right)$ are irreducible components which are contained in homogeneity $\ell$ respectively $\ell+1$. Then the components of the BGG operators $\tilde{D}_{k}$ and $D_{k}$, which map sections of $\mathcal{G} \times{ }_{P} V$ to sections of $\mathcal{G} \times{ }_{P} W$, coincide.

Proof. Consider a section $\alpha \in \Gamma\left(\mathcal{G} \times{ }_{P} V\right)$ and put $\varphi:=L(\alpha) \in \Omega^{k}(M, \mathcal{A M})$. Then $\varphi$ is homogeneous of degree $\geq \ell, \partial^{*}(\varphi)=0$ and $\pi_{H}(\varphi)=\alpha$. By Lemma 3.3.1) we get $d^{\tilde{\nabla}} \varphi=d^{\nabla} \varphi+(-1)^{k} i_{\varphi} \kappa$ and therefore $\partial^{*}\left(d^{\tilde{\nabla}} \varphi\right)=(-1)^{k} \partial^{*}\left(i_{\varphi} \kappa\right)$. By torsion freeness $\kappa$ is homogeneous of degree $\geq 2$, so $i_{\varphi} \kappa$ is homogeneous of degree $\geq \ell+2$. Denoting by $\tilde{Q}$ the operator used in the proof of the theorem, we conclude that $\psi:=(-1)^{k+1} \tilde{Q} \partial^{*}\left(i_{\varphi} \kappa\right)$ 
is homogeneous of degree $\geq \ell+2$. By construction $\partial^{*}(\varphi+\psi)=0, \pi_{H}(\varphi+\psi)=\alpha$, and $\partial^{*}\left(d^{\tilde{\nabla}}(\varphi+\psi)\right)=0$, which implies $\tilde{L}(\alpha)=\varphi+\psi$. Now

$$
d^{\tilde{\nabla}}(\varphi+\psi)=d^{\nabla} \varphi+(-1)^{k} i_{\varphi} \kappa+d^{\tilde{\nabla}} \psi,
$$

and the last two terms are homogeneous of degree $\geq \ell+2$. By homogeneity, these terms cannot contribute to the component of the image under $\pi_{H}$ that we are interested in.

\subsection{On regularity}

To get a complete correspondence to underlying structures, one has to single out regular normal infinitesimal deformations among all normal ones. Here a normal infinitesimal deformation $\varphi \in \Omega^{1}(M, \mathcal{A} M)$ is called regular if $d^{\tilde{\nabla}} \varphi \in \Omega^{2}(M, \mathcal{A} M)$ is homogeneous of degree $\geq 1$. Notice that this condition is vacuous if the geometry corresponds to a $|1|$ grading, and Theorem 3.6 therefore gives a complete description of the formal tangent space to the moduli space of regular normal geometries.

In general, we can first show that trivial infinitesimal deformations of regular normal geometries are regular. Indeed, from Lemma 3.3 (2) we know that for $s \in \Gamma(\mathcal{A} M)$ we have $d^{\tilde{\nabla}} \tilde{\nabla} s=D_{s} \kappa$. If we start from a regular normal geometry, then $\kappa$ is homogeneous of degree $\geq 1$, and by naturality of the fundamental derivative the same is true for $D_{s} \kappa$. Theorem 3.6 now directly implies

Corollary. Let $(p: \mathcal{G} \rightarrow M, \omega)$ be a regular normal parabolic geometry. Then the formal tangent space at the given structure to the moduli space of regular normal geometries is the quotient of the space of all $\alpha \in \Gamma\left(H_{1}\left(T^{*} M, \mathcal{A} M\right)\right)$ such that $d^{\tilde{\nabla}} \tilde{L}(\alpha) \in \Omega^{2}(M, \mathcal{A} M)$ is homogeneous of degree $\geq 1$ by the image of $\tilde{D}_{0}$.

For any concrete choice of structure, the condition on the homogeneity of $d^{\tilde{\nabla}} \tilde{L}(\alpha)$ can be made more explicit by projecting out step by step the lowest possibly non-zero homogeneous components of $d^{\tilde{\nabla}} \circ \tilde{L}$. For structures corresponding to $|2|$-gradings, we can give a nicer description, which will be useful in the examples of Section 4

Proposition. Suppose that $P \subset G$ corresponds to a $|2|$-grading of $\mathfrak{g}$. Then for any regular normal parabolic geometry $(p: \mathcal{G} \rightarrow M, \omega)$ of type $(G, P)$ and any section $\alpha \in \Gamma\left(H_{1}\left(T^{*} M, \mathcal{A} M\right)\right)$ the form $d^{\nabla} \tilde{L}(\alpha) \in \Omega^{2}(M, \mathcal{A} M)$ is homogeneous of degree $\geq 1$ if and only $\tilde{D}_{1}(\alpha)$ is homogeneous of degree $\geq 1$.

Proof. By definition, we have $\tilde{D}_{1}(\alpha)=\pi_{H}\left(d^{\tilde{\nabla}} \tilde{L}(\alpha)\right)$. If $\tilde{D}_{1}(\alpha)$ is homogeneous of degree $\geq 1$, then so is $\tilde{L}\left(\tilde{D}_{1}(\alpha)\right)$, which differs from $d^{\tilde{\nabla}} \tilde{L}(\alpha)$ by a section of $\operatorname{im}\left(\partial^{*}\right)$. Since we are dealing with a $|2|$-grading, any element of $\Lambda^{3} T^{*} M \otimes \mathcal{A}^{M}$ is homogeneous of degree $\geq 1$, and the result follows since $\partial^{*}$ preserves homogeneities.

Since any irreducible component of $H_{2}\left(\mathfrak{p}_{+}, \mathfrak{g}\right)$ is contained in some homogeneous degree, the condition in the proposition simply means that all components of $\tilde{D}_{1}(\alpha)$ in bundles corresponding to irreducible pieces in homogeneity zero have to vanish. 


\subsection{The locally flat case}

As a simple consequence of Theorem 3.6, we can deal with the case of locally flat geometries. The following result was first proved in [5].

Theorem. Let $(p: \mathcal{G} \rightarrow M, \omega)$ be a locally flat parabolic geometry. Then the BGG sequence associated to the adjoint representation is a complex. It can be naturally viewed as a deformation complex, i.e. its homologies in degrees zero and one are the space of infinitesimal automorphisms respectively the formal tangent space to the moduli space of all locally flat parabolic geometries on $M$.

Proof. By local flatness, $\nabla=\tilde{\nabla}$ and this connection is flat, so the twisted de Rham sequence is a complex. This easily implies that $L \circ D=d^{\nabla} \circ L$, so the BGG sequence is also a complex. By Theorem 3.5, the cohomology of this complex in degree zero is isomorphic to the space of infinitesimal automorphisms. For $\alpha \in \Gamma\left(H_{1}\left(T^{*} M, \mathcal{A M}\right)\right)$ with $D_{1}(\alpha)=0$ we have $d^{\nabla} L(\alpha)=L D(\alpha)=0$, so the infinitesimal deformation $L(\alpha)$ does not change the curvature infinitesimally. Since conversely $d^{\nabla} L(\alpha)=0$ clearly implies $D_{1}(\alpha)=0$, we see that the kernel of $D_{1}$ exactly corresponds to the infinitesimal deformations in the subcategory of locally flat geometries. Now the interpretation of the first cohomology follows from Theorem 3.6

\section{Deformation complexes for torsion free geometries}

In the recent joint work [14] with V. Souček, we have developed a theory of subcomplexes in curved BGG sequences. This theory applies to torsion free geometries of certain types. To have interesting examples, one needs assumptions on the structure of the homology group $H_{2}\left(\mathfrak{p}_{+}, \mathfrak{g}\right)$, which forms the degree two part of the adjoint BGG sequence and governs the structure of the harmonic curvature. The main examples of this situation are the ones discussed in [14].

Here we find for all these examples a certain subcomplex in the adjoint BGG sequence (obtained from $d^{\nabla}$ ). Using the results of Section 3, we can show that the first two operators in this subcomplex coincide with their counterparts in the BGG sequence obtained from $d^{\tilde{\nabla}}$. This leads to an interpretation of the subcomplex as a deformation complex in the appropriate subcategory of torsion free geometries.

\subsection{Grassmannian structures}

An almost Grassmannian structure on a manifold $M$ of dimension $2 n$ is essentially given by two auxiliary bundles $E$ and $F$ over $M$ of rank 2 respectively $n$, and an isomorphism $\Phi: E^{*} \otimes F \rightarrow T M$. The bundles $E$ and $F$ are the basic building blocks for bundles over $M$ corresponding to irreducible representations of $P$.

The BGG sequences in this case have triangular shape (see [14, 3.4]). The bundle in degree $k$ of the BGG sequence splits into a direct sum of irreducible subbundles $\mathcal{H}_{p, q}$ with 
$p+q=k$ and $0 \leq p \leq q \leq n$. In particular, the second bundle splits as $\mathcal{H}_{0,2} \oplus \mathcal{H}_{1,1}$, and correspondingly there are two irreducible components in the harmonic curvature. Let us now restrict to the case $n>2$; the case $n=2$ will be discussed in 4.2. The harmonic curvature component in $\Gamma\left(\mathcal{H}_{0,2}\right)$ is called the torsion of the almost Grassmannian structure. Vanishing of this torsion is equivalent to torsion freeness in the sense of $G$-structures, and the corresponding geometries are called Grassmannian rather than almost Grassmannian. The harmonic curvature component in $\mathcal{H}_{1,1}$ is a true curvature. It is shown in [14, Theorem 3.5] that in the case of Grassmannian structures for any $p=0, \ldots, n$ the parts $\mathcal{H}_{p, p} \rightarrow \cdots \rightarrow \mathcal{H}_{p, n}$ and for any $q=0, \ldots, n$ the parts $\mathcal{H}_{0, q} \rightarrow \cdots \rightarrow \mathcal{H}_{q, q}$ are subcomplexes in each BGG sequence.

The representations inducing the bundles in the adjoint BGG sequence are determined in [14, 4.1], where we take $k=\ell=1$. For $j<n$, one obtains $\mathcal{H}_{0, j}=\left(S^{j} E \otimes E^{*}\right)_{0}$ $\otimes\left(\Lambda^{j} F^{*} \otimes F\right)_{0}$, where the subscript 0 denotes the trace free part. In particular $\mathcal{H}_{0,0}=$ $E^{*} \otimes F=T M$, which also follows from Theorem 3.4, and $\mathcal{H}_{0,1}=\mathfrak{s l}(E) \otimes \mathfrak{s l}(F)$. Evidently, $\mathcal{H}_{0, j} M \subset \Lambda^{j}\left(E \otimes F^{*}\right) \otimes\left(E^{*} \otimes F\right)=\Lambda^{J} T^{*} M \otimes T M$. Looking at homogeneities, this implies that the BGG operators $\Gamma\left(\mathcal{H}_{0, j-1}\right) \rightarrow \Gamma\left(\mathcal{H}_{0, j}\right)$ are of first order for all $j=1, \ldots, n-1$. For the last bundle, we get $\mathcal{H}_{0, n}=\left(S^{n+1} E \otimes E^{*}\right)_{0} \otimes \Lambda^{n} F^{*}$, and the last BGG operator $\Gamma\left(\mathcal{H}_{0, n-1}\right) \rightarrow \Gamma\left(\mathcal{H}_{0, n}\right)$ is of second order.

Finally, we need the bundle $\mathcal{H}_{1,1}$ which turns out to be the highest weight part in $\Lambda^{2} E \otimes S^{2} F^{*} \otimes \mathfrak{s l}(F)$. This is contained in $\Lambda^{2} T^{*} M \otimes L(T M, T M)$, so the BGG operator $\Gamma\left(\mathcal{H}_{0,1}\right) \rightarrow \Gamma\left(\mathcal{H}_{1,1}\right)$ is a second order operator.

Theorem. Let $M$ be a Grassmannian manifold of dimension $2 n \geq 6$. Then the subcomplex

$$
0 \rightarrow \Gamma\left(\mathcal{H}_{0,0}\right) \rightarrow \Gamma\left(\mathcal{H}_{0,1}\right) \rightarrow \cdots \rightarrow \Gamma\left(\mathcal{H}_{0, n}\right) \rightarrow 0
$$

of the adjoint BGG sequence is a deformation complex in the subcategory of Grassmannian structures.

Proof. The first two operators in this sequence are just the first two operators in the full adjoint BGG sequence, and from Theorem 3.5 and Lemma 3.6 we conclude that they coincide with their counterparts constructed from $\tilde{\nabla}$ rather than $\nabla$. The statement on the cohomology in degree zero then follows from Theorem 3.4

By Theorem 3.6.2) and since regularity is automatic for $|1|$-gradings, the quotient $\Gamma\left(\mathcal{H}_{0,1}\right) / \operatorname{im}\left(D_{0}\right)$ is isomorphic to infinitesimal deformations of $M$ in the category of almost Grassmannian structures modulo trivial infinitesimal deformations. On the other hand, Theorem 3.6(3) implies that the kernel of $\Gamma\left(\mathcal{H}_{0,1} M\right) \rightarrow \Gamma\left(\mathcal{H}_{0,2} M\right)$ corresponds exactly to those deformations for which the infinitesimal change of torsion is trivial, so these are exactly the infinitesimal deformations in the category of Grassmannian structures.

Remark. (1) For almost Grassmannian structures, the right definition of an infinitesimal deformation is not immediately evident. It is a nice feature of the approach via parabolic geometries and the BGG machinery that it shows that infinitesimal deformations are smooth sections of the bundle $\mathfrak{s l}(E) \otimes \mathfrak{s l}(F)$. This can be seen directly as follows. 
The only part of an almost Grassmannian structure that can be deformed non-trivially is the isomorphism $\Phi: E^{*} \otimes F \rightarrow T M$. Infinitesimally, deformations of this isomorphism are linear maps $E^{*} \otimes F \rightarrow T M$ modulo those which are compatible with $\Phi$. If we use $\Phi$ to convert the target of such a map back to $E^{*} \otimes F$, these are exactly the endomorphisms of $E^{*} \otimes F$ modulo those which are of the form $\varphi \otimes \mathrm{id}_{F}+\mathrm{id}_{E} \otimes \psi$.

(2) By Theorem 3.6, the splitting operator $\tilde{L}: \Gamma\left(\mathcal{H}_{0,1}\right) \rightarrow \Omega^{1}(M, \mathcal{A} M)$ computes the infinitesimal deformation of the canonical Cartan connection caused by an infinitesimal deformation of the underlying structure.

\subsection{The case $n=2$}

In this case, $\operatorname{dim}(M)=4$ and an almost Grassmannian structure is equivalent to a conformal spin structure with split signature $(2,2)$. Basically, this is due to the fact that $S L(4, \mathbb{R})$ is naturally a two-fold covering of $S O(3,3)$. Here the situation is more symmetric than for general Grassmannian structures, and the two components of the harmonic curvature are the self-dual and the anti-self-dual parts of the Weyl curvature. Theorem 3.6 directly leads to a complex

$$
0 \rightarrow \Gamma\left(\mathcal{H}_{0,0}\right) \rightarrow \Gamma\left(\mathcal{H}_{0,1}\right) \rightarrow \Gamma\left(\mathcal{H}_{0,2}\right) \rightarrow 0
$$

inside the BGG sequence obtained from $d^{\tilde{\nabla}}$, and, for anti-self-dual structures, an interpretation as a deformation complex in the category of anti-self-dual conformal structures. This is exactly the split signature version of the complex discussed in 2.3 . However, in this case the second operator (which has order two) differs (tensorially) from its counterpart in the standard adjoint BGG sequence.

\subsection{Quaternionic structures}

An almost quaternionic structure on a smooth manifold of dimension $4 n$ is given by a rank 3 subbundle $Q \subset L(T M, T M)$ which is locally spanned by three almost complex structures $I, J$, and $K=I J=-J I$. However, these local almost complex structures are an additional choice and not an ingredient of the structure. Equivalently, one can view such a structure as a reduction to the structure group $S(G L(1, \mathbb{H}) G L(n, \mathbb{H})) \subset$ $G L(4 n, \mathbb{R})$ of the linear frame bundle. Passing to the two-fold covering $S(G L(1, \mathbb{H}) \times$ $G L(n, \mathbb{H})$ ), one has an equivalent description as an identification of the complexified tangent bundle $T M \otimes \mathbb{C}$ with a tensor product $E \otimes F$, where $E$ has complex rank two and $F$ has complex rank $2 n$. Hence after complexification we are in the same situation as for almost Grassmannian structures with $F$ even-dimensional.

In particular, the BGG sequences have the same shape as in the almost Grassmannian case, and the operators have the same orders. Moreover, after complexification the bundles showing up in each BGG sequence are the same as in the almost Grassmannian case. In particular, there are again two harmonic curvature components and for $n>1$ (the case $n=1$ will be discussed below) one of them is a torsion and the other is a true 
curvature. Vanishing of the torsion is equivalent to torsion freeness in the sense of $G$ structures and the corresponding geometries are referred to as quaternionic rather than almost quaternionic. For quaternionic structures one obtains subcomplexes in all BGG sequences which have the same form as in the Grassmannian case.

Theorem. Let $M$ be a quaternionic manifold of dimension $4 n \geq 8$. Then the subcomplex

$$
0 \rightarrow \Gamma\left(\mathcal{H}_{0,0}\right) \rightarrow \Gamma\left(\mathcal{H}_{0,1}\right) \rightarrow \cdots \rightarrow \Gamma\left(\mathcal{H}_{0, n}\right) \rightarrow 0
$$

of the adjoint $B G G$ sequence is an elliptic complex, which can be naturally interpreted as a deformation complex in the category of quaternionic structures.

Proof. The interpretation as a deformation complex works exactly as in the Grassmannian case. Exactness of the symbol sequence is the special case $k=\ell=1$ of [14, Theorem 4.3].

Describing the bundles which show up in the deformation complex is straightforward, but we do not need this description here. Let us just note the description of the bundle $\mathcal{H}_{0,1}$, whose sections are the infinitesimal deformations of an almost quaternionic structure. For $Q \subset L(T M, T M)$ let $L_{Q}(T M, T M) \subset L(T M, T M)$ denote the subbundle of those endomorphisms which commute with any element of $Q$. Then it turns out that $L(T M, T M) \cong Q \oplus L_{Q}(T M, T M) \oplus \mathcal{H}_{0,1}$ and that $\mathcal{H}_{0,1}$ is isomorphic to the tensor product of $Q$ with the space of trace free elements of $L_{Q}(T M, T M)$.

In the special case $n=1$, an almost quaternionic structure on a four manifold is equivalent to a conformal Riemannian spin structure. As in 4.2, we obtain the deformation complex discussed in 2.3 directly from Theorem 3.6 Again, the second operator in the sequence differs from the one in the standard adjoint BGG sequence. Ellipticity can be easily verified directly.

\subsection{Lagrangean contact structures}

A Lagrangean contact structure on a smooth manifold $M$ of dimension $2 n+1$ is given by a codimension one subbundle $H \subset T M$, which defines a contact structure on $M$, together with a decomposition $H=E \oplus F$ as a direct sum of two Lagrangean (or Legendrean) subbundles. This means that the Lie bracket of two sections of $E$ (respectively $F$ ) is a section of $H$. Since $H$ defines a contact structure, this forces $E$ and $F$ to be of rank at most (and hence exactly) $n$. We will assume $n \geq 2$ throughout.

The form of the BGG sequences is described in [14,3.6]. For $k \leq n$, the bundle in degree $k$ of each BGG sequence splits as $\bigoplus_{p, q} \mathcal{H}_{p, q}$ with $p+q=k$ and $p, q \geq 0$. The decomposition of the bundle in degree $n+k$ has the same form as for degree $n-k+1$. In particular, there are three components in the harmonic curvature. The components in $\Gamma\left(\mathcal{H}_{2,0}\right)$ and $\Gamma\left(\mathcal{H}_{0,2}\right)$ are torsions, which are exactly the obstructions to integrability of the subbundles $E$ and $F$ of $T M$. The component in $\Gamma\left(\mathcal{H}_{1,1}\right)$ is a true curvature. For torsion free geometries, the bundles $E$ and $F$ are integrable, so $M$ locally admits two transversal fibrations onto manifolds of dimension $n+1$ such that the two vertical bundles span 
a contact distribution on $M$ and both are Legendrean. In the torsion free case, there are many subcomplexes in each BGG sequence (see [14, 3.7]). In particular, the bundles $\mathcal{H}_{0, q}$ for $q=0, \ldots, n$ and $\mathcal{H}_{p, 0}$ for $p=0, \ldots, n$ both form subcomplexes.

Specializing to the adjoint BGG sequence, we know from 3.4 that the first bundle $\mathcal{H}_{0,0}$ is the quotient $Q:=T M / H$ of the tangent bundle by the contact subbundle. For $0<j \leq n$ one easily verifies that the bundle $\mathcal{H}_{0, j}$ can be described as follows. Since $E$ and $F$ are Legendrean, the contact structure defines isomorphisms $F \cong E^{*} \otimes Q$. Thus $\Lambda^{j} E^{*} \otimes F \cong \Lambda^{j} E^{*} \otimes E^{*} \otimes Q$, and $\mathcal{H}_{0, j} \subset \Lambda^{j} E^{*} \otimes F$ corresponds to the kernel of the alternation $\Lambda^{j} E^{*} \otimes E^{*} \otimes Q \rightarrow \Lambda^{j+1} E^{*} \otimes Q$. In particular, the operator mapping sections of $\mathcal{H}_{0,0}$ to sections of $\mathcal{H}_{0,1}$ must be of second order, while for $1 \leq j<n$ the operator $\Gamma\left(\mathcal{H}_{0, j}\right) \rightarrow \Gamma\left(\mathcal{H}_{0, j+1}\right)$ is of first order. In the same way, the bundles $\mathcal{H}_{j, 0}$ for $1 \leq j \leq n$ can be described as subbundles in $\Lambda^{j} F^{*} \otimes E$ and one gets the analogous results for the orders of the operators.

Theorem. Let $(M, E, F)$ be a torsion free Lagrangean contact structure. Then the subcomplex

$$
0 \rightarrow \Gamma\left(\mathcal{H}_{0,0}\right) \rightarrow \begin{gathered}
\Gamma\left(\mathcal{H}_{0,1}\right) \\
\left.\oplus \mathcal{H}_{1,0}\right)
\end{gathered} \rightarrow \cdots \rightarrow \begin{gathered}
\Gamma\left(\mathcal{H}_{0, n}\right) \\
\oplus \\
\Gamma\left(\mathcal{H}_{n, 0}\right)
\end{gathered} \rightarrow 0
$$

in the adjoint $B G G$ sequence can be naturally viewed as a deformation complex in the category of torsion free Lagrangean contact structures.

Proof. From Theorem 3.5 and Lemma 3.6 we see that the first two operators in this complex coincide with their counterparts in the BGG sequence obtained from $d^{\tilde{\nabla}}$. Since the bundles $\mathcal{H}_{2,0}, \mathcal{H}_{1,1}$, and $\mathcal{H}_{0,2}$ are all contained in positive homogeneity degrees, normal infinitesimal deformations are automatically regular by Proposition 3.7 . Now the interpretation as a deformation complex works as for Grassmannian structures.

Note that the deformation complex cannot be elliptic or subelliptic, since $Q$ is a real line bundle, while all other bundles showing up in the subcomplex have even rank.

\subsection{CR structures}

This case is closely parallel to the case of Lagrangean contact structures. The geometries in question are non-degenerate almost CR structures of hypersurface type, which satisfy the partial integrability condition, a weakening of the usual integrability condition for CR structures (see [10, 4.15]). Compared to the case of Lagrangean contact structures, one only replaces the decomposition of the contact subbundle (which can be interpreted as an almost product structure) by an almost complex structure $J$ on the contact subbundle $H$. The condition that the two subbundles are Legendrean corresponds to the partial integrability condition for almost $\mathrm{CR}$ structures. The complexification $H \otimes \mathbb{C}$ splits as $H^{1,0} \oplus H^{0,1}$ into holomorphic and anti-holomorphic part, so on this level the picture is parallel to the Lagrangean contact case. 
In particular, complex BGG sequences have the same form as for Lagrangean contact structures. However, BGG sequences corresponding to real representations without an invariant complex structure (like the adjoint representation) are different. They are obtained by "folding" a complex BGG pattern (see [14, 3.8]). In particular, there are only two irreducible components in the harmonic curvature. One of these components is a torsion (corresponding to the two torsions for Lagrangean contact structures), while the other is a curvature. The torsion is a multiple of the Nijenhuis tensor, so the torsion free geometries are exactly the CR structures (see [10, 4.16]). For CR structures, one obtains many subcomplexes in BGG sequences (see [14, Theorem 3.8]).

In the notation of [14, 3.8], there is a subcomplex in the adjoint BGG sequence which starts at $\mathcal{H}_{0,0}$. It has the form

$$
0 \rightarrow \Gamma\left(\mathcal{H}_{0,0}\right) \rightarrow \Gamma\left(\mathcal{H}_{1,0}\right) \rightarrow \cdots \rightarrow \Gamma\left(\mathcal{H}_{n, 0}\right) \rightarrow 0
$$

and apart from $\mathcal{H}_{0,0}=Q:=T M / H$, all the bundles $\mathcal{H}_{j, 0}$ in the sequence are complex vector bundles. To identify them, one passes to the complexification and uses the results from the Lagrangean contact case with $E$ and $F$ replaced by $H^{1,0}$ and $H^{0,1}$. In particular, $\mathcal{H}_{j, 0} \otimes \mathbb{C} \subset L_{\mathbb{C}}\left(\Lambda^{j}(H \otimes \mathbb{C})^{*}, H \otimes \mathbb{C}\right)$ and the components are singled out by their complex (anti-)linearity properties. For example, $\mathcal{H}_{0,1} \otimes \mathbb{C}$ is contained in $L\left(H^{1,0}, H^{0,1}\right) \oplus L\left(H^{0,1}, H^{1,0}\right)$, which exactly means that $\mathcal{H}_{0,1}$ consists of conjugate linear maps $H \rightarrow H$. A conjugate linear map $\varphi$ lies in $\mathcal{H}_{0,1}$ if and only if the corresponding bilinear map $H \times H \rightarrow Q$ is symmetric. In particular, conjugate linear maps are exactly infinitesimal deformations of the almost complex structure $J$ (which is the only deformable ingredient in the structure) and the symmetry condition takes care of partial integrability. As before we deduce:

Theorem. Let $(M, H, J)$ be a non-degenerate CR structure of hypersurface type. Then the subcomplex

$$
0 \rightarrow \Gamma\left(\mathcal{H}_{0,0}\right) \rightarrow \Gamma\left(\mathcal{H}_{1,0}\right) \rightarrow \cdots \rightarrow \Gamma\left(\mathcal{H}_{n, 0}\right) \rightarrow 0
$$

in the adjoint BGG sequence can be naturally interpreted as a deformation complex in the category of CR structures.

Remark. This deformation complex has been found earlier (by ad hoc methods) and successfully applied to the deformation theory of strictly pseudoconvex compact CR manifolds. The part starting from $\mathcal{H}_{1,0} M$ is used in the work of T. Akahori in the case $n \geq 3$ (see e.g. [1]). The full complex was constructed in [2] for $n=2$. Since the first bundle in the complex is a real line bundle while all others are complex vector bundles, there is again no hope for the whole complex to be elliptic or subelliptic. Nonetheless, for some of the operators in the complex one can prove subelliptic estimates (in the strictly pseudoconvex case), which play a crucial role in the applications to deformation theory. 


\subsection{Quaternionic contact structures}

These geometries are given by certain codimension three subbundles in the tangent bundles of manifolds of dimension $4 n+3$. Recall first that for $p+q=n$, there is (up to isomorphism) a unique quaternionic Hermitian form of signature $(p, q)$ on $\mathbb{H}^{n}$. The imaginary part of this form is a skew symmetric bilinear map $\mathbb{H}^{n} \times \mathbb{H}^{n} \rightarrow \mathrm{im}(\mathbb{H})$. If we put $\mathfrak{g}_{1}:=\mathbb{H}^{n}$ and $\mathfrak{g}_{2}:=\operatorname{im}(\mathbb{H})$, this imaginary part makes $\mathfrak{g}_{1} \oplus \mathfrak{g}_{2}$ into a nilpotent graded Lie algebra, called the quaternionic Heisenberg algebra of signature $(p, q)$. Since the forms of signature $(p, q)$ and $(q, p)$ differ only by sign, we may assume $p \geq q$. Similarly, one may look at the algebra of split quaternions, for which there is a unique Hermitian form in each dimension. Correspondingly, one obtains a unique split quaternionic Heisenberg algebra of dimension $4 n+3$ for each $n \geq 1$.

Recall that if $H \subset T M$ is a subbundle in the tangent bundle of a smooth manifold $M$, then the Lie bracket of vector fields induces a tensorial map $\mathcal{L}: H \times H \rightarrow T M / H=: Q$. For any $x \in M$ we put $\operatorname{gr}_{1}\left(T_{x} M\right)=H_{x}$ and $\operatorname{gr}_{2}\left(T_{x} M\right):=Q_{x}$. Then we can view $\mathcal{L}$ as defining on each of the spaces $\operatorname{gr}\left(T_{x} M\right)=\operatorname{gr}_{1}\left(T_{x} M\right) \oplus \operatorname{gr}_{2}\left(T_{x} M\right)$ the structure of a nilpotent graded Lie algebra. A quaternionic contact structure of signature $(p, q)$ on a smooth manifold $M$ of dimension $4(p+q)+3$ is a smooth subbundle $H \subset T M$ of corank 3 such that for each $x \in M$ the nilpotent graded Lie algebra $\operatorname{gr}\left(T_{x} M\right)$ is isomorphic to the quaternionic Heisenberg algebra of signature $(p, q)$. A split quaternionic contact structure on a smooth manifold of dimension $4 n+3$ is defined similarly using the split quaternionic Heisenberg algebra.

For $n=1$ we have $\operatorname{dim}(M)=7$ and there is only one possible signature. It turns out that both the quaternionic and the split quaternionic Heisenberg algebra are rigid in this case. Moreover, corank three distributions defining quaternionic and split quaternionic contact structures are the two generic types of rank 4 distributions in dimension 7. In particular, a generic real hypersurface in a two-dimensional (split) quaternionic vector space carries a (split) quaternionic contact structure.

For $n>1$, there are no generic distributions of rank $4 n$ in manifolds of dimension $4 n+3$, but it is known from the works of O. Biquard (see [3, 4]) that there are many examples of quaternionic contact structures of signature $(n, 0)$.

For all these structures, the BGG sequences have the same form (see [14, 3.9]). For $k=0, \ldots, 2 n+1$ the bundle in degree $k$ splits into a direct sum of bundles $\mathcal{H}_{p, q}$ with $p+q=k$ and $p \geq q$, and the bundle in degree $2 n+1+k$ decomposes in the same way as the one in degree $2 n+2-k$. In particular, in degree two we obtain two irreducible components $\mathcal{H}_{2,0}$ and $\mathcal{H}_{1,1}$. The harmonic curvature component having values in the bundle $\mathcal{H}_{2,0}$ of the adjoint $\mathrm{BGG}$ sequence is a torsion, while the one having values in $\mathcal{H}_{1,1}$ is a curvature. For $n=1$, one obtains a subcategory of torsion free (split) quaternionic contact structures. However, for $n>1$, the bundle $\mathcal{H}_{2,0}$ is contained in homogeneity zero, so vanishing of the corresponding harmonic curvature component is forced by regularity, and any (split) quaternionic contact structure is automatically torsion free.

By [14, Theorem 3.10] there are a number of subcomplexes in each BGG sequence for a manifold endowed with a torsion free (split) quaternionic contact structure. In particular, the bundles $\mathcal{H}_{p, 0}$ with $p=0, \ldots, 2 n+1$ form a subcomplex. For the adjoint BGG 
sequence, one verifies directly that the operator $\Gamma\left(\mathcal{H}_{n, 0}\right) \rightarrow \Gamma\left(\mathcal{H}_{n+1,0}\right)$ is of second order, while all other operators in the subcomplex are of first order.

Theorem. Let $M$ be a smooth manifold of dimension $4 n+3 \geq 11$ endowed with a quaternionic contact structure or split quaternionic contact structure. Then the subcomplex

$$
0 \rightarrow \Gamma\left(\mathcal{H}_{0,0}\right) \rightarrow \Gamma\left(\mathcal{H}_{1,0}\right) \rightarrow \cdots \rightarrow \Gamma\left(\mathcal{H}_{2 n+1,0}\right) \rightarrow 0
$$

of the adjoint $B G G$ sequence can be naturally interpreted as a deformation complex in the category of (split) quaternionic contact structures.

Proof. By Theorem 3.5 and Lemma 3.6 the first two operators in this sequence coincide with their counterparts obtained from $d^{\tilde{\nabla}}$. Using Proposition 3.7 we conclude that the kernel of the operator $\mathcal{H}_{1,0} \rightarrow \mathcal{H}_{2,0}$ exactly corresponds to regular normal deformations. The interpretation as a deformation complex then works as before.

Remark. The situation in the seven-dimensional case is not completely clear. The problem here is that the operator $\mathcal{H}_{1,0} \rightarrow \mathcal{H}_{2,0}$ in the adjoint BGG sequence is of second order. It seems that the two operators obtained from $d^{\nabla}$ respectively from $d^{\tilde{\nabla}}$ differ (tensorially) from each other. Therefore, there is no direct way to relate the BGG sequence based on $d^{\nabla}$ (for which we can prove the existence of the relevant subcomplex) to the one based on $d^{\tilde{\nabla}}$ (for which we have the interpretation in terms of infinitesimal deformations).

Acknowledgments. This work was supported by project P15747-N05 of the "Fonds zur Förderung der wissenschaftlichen Forschung" (FWF). Discussions with D. Calderbank, M. Eastwood, and R. Gover were very helpful. This paper grew out of the joint work [14] with V. Souček and I particularly want to thank him for many discussions. Finally, I would like to thank the referee for several interesting comments and suggestions.

\section{References}

[1] Akahori, T.: The new estimate for the subbundles $E_{j}$ and its application to the deformation of the boundaries of strongly pseudoconvex domains. Invent. Math. 63, 311-334 (1981) Zbl 0496.32015 MR 0610542

[2] Akahori, T., Garfield, P. M., Lee, J. M.: Deformation theory of 5-dimensional CR structures and the Rumin complex. Michigan Math. J. 50, 517-549 (2002) Zbl 1065.32018 MR 1935151

[3] Biquard, O.: Métriques d'Einstein asymptotiquement symétriques. Astérisque 265 (2000) Zbl 0967.53030 MR 1760319

[4] Biquard, O.: Quaternionic contact structures. In: Quaternionic Structures in Mathematics and Physics (Rome, 1999) (electronic), Univ. Studi Roma, "La Sapienza", 23-30 (1999) Zbl 0993.53017 MR 1848655

[5] Calderbank, D. M. J., Diemer, T.: Differential invariants and curved Bernstein-GelfandGelfand sequences. J. Reine Angew. Math. 537, 67-103 (2001) Zbl 0985.58002 MR 1856258

[6] Čap, A.: Correspondence spaces and twistor spaces for parabolic geometries, J. Reine Angew. Math. 582, 143-172 (2005) Zbl 1075.53022 MR 2139714 
[7] Čap, A.: Two constructions with parabolic geometries. Rend. Circ. Mat. Palermo Suppl. 79, 11-37 (2006) Zbl 1120.53013 MR 2287124

[8] Čap, A., Gover, A. R.: Tractor calculi for parabolic geometries. Trans. Amer. Math. Soc. 354, 1511-1548 (2002), Zbl 0997.53016 MR 1873017

[9] Čap, A., Gover, A. R.: Standard tractors and the conformal ambient metric construction. Ann. Global Anal. Geom. 24, 231-259 (2003) Zbl 1039.53021 MR 1996768

[10] Čap, A., Schichl, H.: Parabolic geometries and canonical Cartan connections. Hokkaido Math. J. 29, 453-505 (2000), Zbl 0996.53023 MR 1795487

[11] Čap, A., Slovák, J.: Weyl structures for parabolic geometries. Math. Scand. 93, 53-90 (2003) Zbl 1076.53029 MR 1997873

[12] Čap, A., Slovák, J.: Parabolic Geometries I: Background and General Theory. Book in preparation

[13] Čap, A., Slovák, J., Souček, V.: Bernstein-Gelfand-Gelfand sequences. Ann. of Math. 154, 97-113 (2001), Zbl pre01657117 MR 1847589

[14] Čap, A., Souček, V.: Subcomplexes in curved BGG sequences. Preprint ESI 1683 (2005); http://www.esi.ac.at

[15] Gasqui, J., Goldschmidt, H.: Déformations infinitésimales des structures conformes plates. Progr. Math. 52, Birkhäuser (1984) Zbl 0585.53001 MR 0776970

[16] Itoh, M.: Moduli of half conformally flat structures. Math. Ann. 296, 687-708 (1993) Zbl 0788.58011 MR 1233492

[17] King, A. D., Kotschick, D.: The deformation theory of anti-self-dual conformal structures. Math. Ann. 294, 591-609 (1992) Zbl 0765.58005 MR 1190446

[18] Kostant, B.: Lie algebra cohomology and the generalized Borel-Weil theorem. Ann. of Math. 74, 329-387 (1961), Zbl 0134.03501 MR 0142696

[19] Yamaguchi, K.: Differential systems associated with simple graded Lie algebras. Adv. Stud. Pure Math. 22, 413-494 (1993) Zbl 0812.17018 MR 1274961 